Neurogastroenterol Motil. Author manuscript; available in PMC 2012 December 01.

Published in final edited form as:

Neurogastroenterol Motil. 2011 December ; 23(12): 1132-1139. doi:10.1111/j.1365-2982.2011.01796.x.

\title{
The anxiolytic effect of Bifidobacterium longum NCC3001 involves vagal pathways for gut-brain communication
}

\author{
P. BERCIK ${ }^{\star}$, A. J. PARK ${ }^{\star}$, D. SINCLAIR ${ }^{\star}$, A. KHOSHDEL ${ }^{*}$, J. LU ${ }^{\star}$, X. HUANG ${ }^{\star}$, Y. DENG ${ }^{\star}$, P. A. \\ BLENNERHASSETT ${ }^{\star}$, M. FAHNESTOCK ${ }^{\dagger}$, D. MOINE ${ }^{\ddagger}$, B. BERGER ${ }^{\ddagger}$, J. D. HUIZINGA ${ }^{\star}, W$. \\ KUNZE $^{*}$, P. G. MCLEAN§, G. E. BERGONZELLI§, S. M. COLLINS ${ }^{\star}$, and E. F. VERDU*
}

*Department of Medicine, Farncombe Family Digestive Health Research Institute, McMaster University, Hamilton, ON, Canada tDepartment of Psychiatry \& Behavioural Neurosciences, McMaster University, Hamilton, ON, Canada ‡Bioanalytical Science Department, Nestlé Research Center, Lausanne, Switzerland §Digestive Health, Nutrition and Health Department, Nestlé Research Center, Lausanne, Switzerland

\section{Abstract}

Background-The probiotic Bifidobacterium longum NCC3001 normalizes anxiety-like behavior and hippocampal brain derived neurotrophic factor (BDNF) in mice with infectious colitis. Using a model of chemical colitis we test whether the anxiolytic effect of $\mathrm{B}$. longum involves vagal integrity, and changes in neural cell function.

Methods-Mice received dextran sodium sulfate (DSS, 3\%) in drinking water during three 1week cycles. Bifidobacterium longum or placebo were gavaged daily during the last cycle. Some mice underwent subdiaphragmatic vagotomy. Behavior was assessed by step-down test, inflammation by myeloperoxidase (MPO) activity and histology. BDNF mRNA was measured in neuroblastoma SH-SY5Y cells after incubation with sera from B. longum- or placebo-treated mice. The effect of B. longum on myenteric neuron excitability was measured using intracellular microelectrodes.

Key Results-Chronic colitis was associated with anxiety-like behavior, which was absent in previously vagotomized mice. B. longum normalized behavior but had no effect on MPOactivity or histological scores. Its anxiolytic effect was absent in mice with established anxiety that were

Address for Correspondence: Elena F Verdu, MD, PhD, Farncombe Family Digestive Health Research Institute, McMaster University, HSC 3N8, Hamilton, Ontario, Canada L8S 4K1. Tel: 1 (905) 525 9140/Ext 21952; fax: 1 (905) 522 3454; verdue@ mcmaster.ca. AUTHOR CONTRIBUTIONS

PB performed the research, analyzed results, contributed to scientific discussion and wrote the paper. AJP analyzed results and wrote the paper. JL, XH, DS, YD, PAB, AK, DM and BB performed and analyzed experiments. JDH and WK contributed to scientific discussion and edited manuscript. MF contributed essential reagents and cell lines. SMC contributed to scientific discussion and edited manuscript. GEB and PGM contributed essential reagents (Bifidobacterium longum), contributed to scientific discussion and edited manuscript. EFV conceived, performed the research, analyzed results and wrote the paper.

CONFLICT OF INTEREST

Drs. Berger, Moines, Bergonzelli and McLean are employees of Nestle, Switzerland. The remaining authors declare no conflict of interest.

DISCLOSURES

The authors confirm they had complete access to the data that supports the publication, as the work was entirely performed at McMaster University. 
vagotomized before the third DSS cycle. B. longum metabolites did not affect BDNF mRNA expression in SH-SY5Y cells but decreased excitability of enteric neurons.

Conclusions \& Inferences-In this colitis model, anxiety-like behavior is vagally mediated. The anxiolytic effect of B. longum requires vagal integrity but does not involve gut immunomodulation or production of BDNF by neuronal cells. As B. longum decreases excitability of enteric neurons, it may signal to the central nervous system by activating vagal pathways at the level of the enteric nervous system.

\section{Keywords}

behavior; brain-derived neurotrophic factor; gut-brain axis; probiotics; vagus

\section{INTRODUCTION}

Psychiatric co-morbidities such as anxiety and depression are common in patients with chronic gut disorders, including those with overt inflammatory conditions of the gastrointestinal (GI) tract, such as inflammatory bowel disease, ${ }^{1}$ and those with low-grade gut inflammation as found in irritable bowel syndrome. ${ }^{2}$ Animal models have shown that both acute and chronic GI infection and inflammation result in changes in host behavior and central nervous system (CNS) biochemistry, ${ }^{3-6}$ but the precise mechanisms of this altered gut- brain communication are not completely understood. Possible candidate routes include neural and humoral inputs from the periphery to the CNS.

Animal studies investigating the effect of systemic inflammation have shown that proinflammatory cytokines can alter tryptophan metabolism, increasing levels of kynurenin, which is known to induce anxiety-like behavior in a dose-dependent manner. ${ }^{7}$ Studies focused on the GI tract have demonstrated that the development of anxiety-like behavior during the acute phase of infection with Campylobacter pylori is associated with activation of vagal pathways. ${ }^{4}$ We have recently shown that chronic colitis, caused by a non-invasive parasite Trichuris muris, resulted in decreased hippocampal brain derived neurotrophic factor (BDNF) levels and abnormal mouse behavior, which was independent of vagal integrity. ${ }^{6}$ Administration of the probiotic Bifidobacterium longum NCC3001 normalized the altered behavior and CNS changes induced by chronic infection, although it did not affect the peripheral cytokine levels or the kynurenin/tryptophan ratio. Thus, the mechanism(s) through which this probiotic affects the gut-brain axis remain unclear.

This study explores the mechanisms of gut-brain communication underlying the effect of $B$. longum on behavior during non-infectious, chronic, low-grade gut inflammation. We assessed mouse behavior following repeated administration of low dose dextran sodium sulfate (DSS), before and after treatment with this probiotic bacterium. A non-infectious chemical model was chosen to avoid the confounding factors related to ongoing active nematode infection. To test the hypothesis that the anxiolytic effect of $B$. longum on behavior involves neural pathways, a group of mice was subjected to vagotomy and pyloroplasty after induction of anxiety-like behavior by chronic DSS. In separate experiments, we treated myenteric neurons in situ with B. longum fermented medium or non-fermented medium (control medium), to determine whether bacterial products 
generated during fermentation can directly alter the excitatory properties of enteric nerves. To investigate the possibility that $B$. longum can up-regulate production of neurotrophins, we incubated human SH-SY5Y neuroblastoma cells with serum of mice treated with this probiotic and assessed BDNF mRNA by real-time RT-PCR. Our results show that in this model of chronic colitis, $B$. longum gut-brain communication involves vagal pathways. Our in vitro results suggest that $B$. longum fermentation products do not directly affect BDNF production by a neural cell line but modify the functional status of enteric neurons in the gut.

\section{MATERIALS AND METHODS}

\section{Animals}

Male AKR mice were purchased from Jackson Laboratories (Bar Harbor, ME, USA) at 6-8 weeks of age and housed in a specific pathogen-free unit at the McMaster University Central Animal Facility. All experiments were conducted in accordance with the guidelines of the Canadian Council on Animal Care and received approval from the McMaster University Animal Research Ethics Board. Mice were allowed to acclimatize for 1 week prior to commencement of the study. All mice were monitored daily following surgery for changes in weight and body appearance.

\section{Study design}

Chronic colitis was induced by administering DSS (ICN Biomedicals Inc., Irvine, CA, USA) in drinking water in three 7-day cycles $\left(3.5,3.0\right.$ and $3.0 \% \mathrm{wt} \mathrm{vol}^{-1}$ for cycles 1,2 and 3, respectively) with a 5 day recovery period after each cycle when the mice drank normal water only. Age-matched control mice received normal drinking water only throughout the entire study. Probiotic-treated mice were daily gavaged intragastrically with $100 \mu \mathrm{L}$ of $1 \times$ E10 cfu B. longum per $\mathrm{mL}$ in fermented medium (FM) during the third cycle of DSS and for additional 7 days after DSS administration. Placebo controls were gavaged with $100 \mu \mathrm{L}$ of non-fermented medium [control medium (CM)].

To determine the role of the vagus nerve in gut brain communication in DSS colitis, one group of mice was vagotomized before the induction of colitis and allowed to recover for 7 days, after which three cycles of DSS were administered. Behavior was assessed at the end of the third cycle.

To investigate the mechanismof communication of $B$. longumin the DSS model, the same chronic inflammation and probiotic/placebo treatment protocol wasused for a separate group ofmice that underwent subdiaphragmatic vagotomy and surgical pyloroplasty $48 \mathrm{~h}$ after the termination of the second cycle of DSS. Behavior was assessed after the third DSS cycle, on day 14 of the probiotic/placebo treatment using step-downand light preference tests.Themicewere sacrificed $24 \mathrm{~h}$ later, and colonic tissues were taken for histology and myeloperoxidase assay (MPO). Additional healthy mice (without DSS) were treated with $B$. longum for 2 weeks, 3 weeks after subdiaphragmatic vagotomy or sham surgery. 


\section{Subdiaphragmatic vagotomy $(\mathrm{Vx})$}

Mice were anesthetized with ketamine (150 $\mathrm{mg} \mathrm{kg}^{-1}$, i.p.; Bimeda-MTC, Cambridge, ON, Canada) and xylazine (10 $\mathrm{mg} \mathrm{kg}^{-1}$, i.p.; Bayer Health Care, Toronto, ON, Canada) via intraperitoneal injection. The abdomen was sterilized and a midline incision was made to expose the gastroesophageal junction. The ventral and dorsal truncal branches of the vagus nerve were located under a stereodissection microscope and transected. As vagotomy can result in pyloric stenosis, all vagotomized and control mice underwent surgical pyloroplasty. The incisions were closed by suture and mice treated with intramuscular antibiotic (Baytril ${ }^{\circledR}$, Bayer Health Care). The completeness of vagotomy was verified at sacrifice by inspection of vagal nerve endings using microscopy.

\section{Behavior assessment}

The step-down test is a well-characterized method to quantify anxiety-like behavior in mice.

${ }^{8}$ Briefly, the mice were individually placed in the center of a raised platform $(10 \mathrm{~cm}$ diameter, $4 \mathrm{~cm}$ high). The latency for all four paws to step down to a black Plexiglas tabletop was measured (maximum duration $5 \mathrm{~min}$ ).

\section{Histology and MPO activity}

Formalin-fixed tissues from the distal colon were stained with hematoxylin and eosin. The slides were scored by a blinded observer using a previously established protocol. ${ }^{9}$ Separate tissue samples were taken to assess granulocytic infiltration via MPO activity as previously described. ${ }^{10}$

\section{Bacterial culture}

Bifidobacterium longum NCC3001 (ATCC BAA-999, initially provided by Morinaga, Tokyo, Japan), was obtained from the Nestlé Culture Collection (Lausanne, Switzerland) and grown for $24 \mathrm{~h}$ under anaerobic conditions in DE Man-Rogosa-Sharpe broth containing $0.5 \%$ cysteine and further processed as previously described. ${ }^{11}$ Briefly, bacterial cells were pelleted by centrifugation for $15 \mathrm{~min}$ at $5000 \mathrm{~g}$ at $4{ }^{\circ} \mathrm{C}$, further resuspended at a concentration of $10^{10} \mathrm{~mL}^{-1}$ in their fermented medium (FM), and kept in frozen aliquots until use. For electrophysiology experiments, FM was filtered through $0.2 \mu \mathrm{mol} \mathrm{L}-1$ filter to obtain bacteria-free medium.

\section{Cell culture}

Human SH-SY5Y neuroblastoma cells (ATCC CRL-2266) were cultured as previously described $^{12}$ in Dulbecco's modified Eagle's medium: Nutrient Mixture F12 (DMEM/F12) supplemented with $10 \%$ fetal bovine serum, $2 \mathrm{mmol} \mathrm{L}^{-1} \mathrm{~L}$-glutamine and $1 \%$ penicillin/ streptomycin (Invitrogen, Burlington, ON, Canada) at $37^{\circ} \mathrm{C}$ in a saturated humidity atmosphere containing 5\% $\mathrm{CO} 2$. To initiate differentiation, $3.25 \times 10^{5}$ cells were seeded in each well of a six-well tissue culture plate (Sarstedt, Montreal, QC, Canada), incubated with the above medium for $24 \mathrm{~h}$, and then incubated with DMEM/F12 containing $10 \mu \mathrm{mol} \mathrm{L}-1$ all-trans retinoic acid (Sigma, Oakville, ON, Canada), 1\% N-2 supplement (Invitrogen), 2 mmol L ${ }^{-1} \mathrm{~L}$-glu, $1 \%$ Pen/Strep and $0.5 \%$ FBS for 6 days with daily replacement of one-half of the medium. Differentiated cells were washed three times and then incubated for $48 \mathrm{~h}$ 
with DMEM/F12 containing $100 \mu \mathrm{L}$ of sera from healthy mice treated with $B$. longum or placebo for 14 days. Nicotine $\left(0.5 \mathrm{mmol} \mathrm{L}^{-1}\right.$, Sigma) was been used as positive control, as it has been shown to promote the release of BDNF from human SH-SY5Y neuroblastoma cells. $^{13}$

\section{RNA isolation and real time RT-PCR}

Total RNA was isolated from the neuroblastoma cells using RNeasy Mini Kit (Qiagen, Mississauga, ON, Canada) and treated with RNase-free DNase (Qiagen) to remove contaminating genomic DNA. Standard reverse transcription was performed using $1 \mu \mathrm{g}$ of RNA and M-MLV reverse-transcriptase (Invitrogen). PCR efficiency and optimal annealing temperature for each of the primer pairs was tested via standard curve and thermal gradient experiments, respectively, using a MyiQ2 Real-Time PCR Detection System (BioRad, Mississauga, ON, Canada). The PCR condition used were as follows: $95^{\circ} \mathrm{C}$ for $30 \mathrm{~s}$, followed by 35 cycles of $95^{\circ} \mathrm{C}$ for $5 \mathrm{~s}$ and $60^{\circ} \mathrm{C}$ for $10 \mathrm{~s}$. Amplification was performed in triplicate $10 \mu \mathrm{L}$ reactions and normalized to glyceraldehyde-3-phosphate dehydrogenase (GAPDH) expression. The product size was confirmed by gel electrophoresis and specificity of the amplification was tested by performing melt-curve analysis for all reactions. BDNF primer sequence: forward 5'-AAA CAT CCG AGG ACA AGG TG; reverse 5' -AGA AGA GGA GGC TCC AAA GC. GAPDH primer sequence: forward 5' -CCA TGG AGA AGG CTG GGG; reverse $5^{\prime}$-CAA AGT TGT CAT GGA TGA CC. Gene expression was analyzed using the $2-\Delta \Delta \mathrm{Ct}$ method.

\section{Electrophysiology}

The excitability of the murine myenteric plexus neurons was tested in response to $B$. longum FM or CM using intracellular microelectrode recordings in situ. Briefly, ileal tissue was excised from 6- to 8-week-old male AKR mice (Jackson Laboratories) following cervical dislocation. Tissues were immediately placed in a physiological buffer (Krebs solution: $118.1 \mathrm{mmol} \mathrm{L}^{-1} \mathrm{NaCl}, 4.8 \mathrm{mmol} \mathrm{L}^{-1} \mathrm{KCl}, 1.2 \mathrm{mmol} \mathrm{L}^{-1} \mathrm{MgSO}_{4}, 25 \mathrm{mmol} \mathrm{L}^{-1} \mathrm{NaHCO}_{3}$, $1.0 \mathrm{NaH}_{2} \mathrm{PO}_{4}, 2.5 \mathrm{mmol} \mathrm{L}^{-1} \mathrm{CaCl}_{2}, 11.1 \mathrm{mmol} \mathrm{L}^{-1}$ glucose) and oxygenated (95\% $\mathrm{O}^{2}: 5 \%$ $\mathrm{CO} 2$ ). The tissue was cut along the mesenteric border, pinned with the mucosa facing up, and treated with $2 \mu \mathrm{mol} \mathrm{L}^{-1} 1: 1$ nicardipine: scopolamine to prevent tissue contraction. Following removal of the mucosa, submucosa, deep muscular plexus, and circular muscle layers, the longitudinal muscle myenteric plexus preparation was placed on the recording chamber of a high powered inverted microscope (Zeiss Axiovert S100 TV) and allowed to equilibrate for up to $90 \mathrm{~min}$ while continuously being perfused $\left(4 \mathrm{~mL} \mathrm{~min}{ }^{-1}\right)$ with the Krebs solution at $37{ }^{\circ} \mathrm{C}$. The tissue was then perfused with either $\mathrm{CM}$ or B. longum FM diluted five times in Krebs buffer, or Krebs buffer alone. Recordings were obtained immediately after exposure using a MultiClamp 700B amplifier and Digidata 1322A acquisition system (Axon Instruments, Weatherford, TX, USA). Using this method, both motor and sensory nerves will be recorded from. The electrodes used for recording were conventional borosilicate glass micropipettes filled with $1 \mathrm{~mol} \mathrm{~L}^{-1} \mathrm{KCl}$ (fire polished; length: $7 \mathrm{~cm}$; O.D.: $1.5 \mathrm{~mm}$; I.D.: $0.86 \mathrm{~mm}$; fabricated to yield resistances between 100 and $200 \mathrm{M} \Omega$ ). Neuronal excitability (electro-responsiveness) was assessed by injecting a $500 \mathrm{~ms}$ depolarizing current with magnitude equal to rheobase plus $50 \mathrm{pA}$ once every $10 \mathrm{~s}$. Electro-responsiveness is 
expressed as the number of action potentials a neuron fires in response to a depolarizing current injection with supra-threshold intensity.

\section{Molecular detection of B. longum NCC3001 in caecal and fecal samples}

DNA was extracted from ceacal and fecal samples using the QIAamp DNA Stool mini kit (Qiagen, Hilden, Germany). The presence of B. longum DNA in these extracts was detected by PCR with strain-specific primers, as previously described. ${ }^{14}$ Products were analyzed on microfluidic chips (LabChip GXII, Caliper Life Sciences, Oftringen, Switzerland).

\section{Data analysis}

Statistical analysis was completed using GraphPad Prism software (GraphPad Software, La Jolla, CA, USA). Data are presented as individual dot plots and mean, or as mean \pm SD. Most comparisons were performed by unpaired student's $t$-tests. For data sets that were nonGaussian or based on a score, data was compared using non-parametric tests. A $P$ value of $<0.05$ was considered significant.

\section{RESULTS}

\section{Effect of $B$. longum on inflammation-induced anxiety-like behavior}

Mice treated with DSS displayed anxiety-like behavior compared with controls. The latency to step down from the elevated platform in DSS-treated mice was twofold greater than in mice that received water only. Administration of $B$. longum to DSS-treated mice not only reversed their altered behavior relative to placebo-treated animals, but mice stepped down from the platform almost twofold faster than healthy controls (Fig. 1).

\section{Effect of $B$. longum on chronic colitis}

Administration of DSS induced chronic, low-grade colonic inflammation characterized by low-to-moderate infiltration of the mucosal and submucosal compartments by mononuclear cells. MPO activity was mildly but significantly elevated in DSS-treated mice.

Administration of $B$. longum had no effect on the level of chronic inflammatory infiltrate orMPOactivity (Fig. 1).

\section{Role of the vagus nerve in DSS-colitis induced anxiety-like behavior}

To evaluate the role of the vagus nerve in DSS colitis-induced anxiety-like behavior, vagotomy was performed before the first cycle of DSS. Sham-operated mice treated with DSS displayed delayed latency to step down from the elevated platform compared with sham-operated mice that received water only (Fig. 2). However, vagotomized mice treated with DSS stepped down with similar latency as mice treated with water only.

\section{Effect of vagotomy on B. longum-induced behavior and inflammation}

When vagotomy was performed in mice with established anxiety and before the third cycle of DSS, mice maintained longer latency to step down from the platform than vagotomized mice that received water only. More importanly, the ability of B. longum to modify the DSSinduced anxiety-like behavior was lost in mice that were vagotomized before the third cycle 
of DSS (Fig. 3A). Histological scores and MPO activity levels were similar in vagotomized mice treated with DSS and either placebo or B. longum (Fig. 3B,C).

In additional experiments, $B$. longum was administered to healthy mice with or without vagotomy. Healthy $B$. longum-treated mice stepped down faster than placebo-treated mice. This effect was not observed in vagotomized mice (Fig. 3D).

\section{Effect of $B$. longum fermentation products on BDNF mRNA expression}

Neuroblastoma cells incubated for $48 \mathrm{~h}$ with sera of healthy mice treated with $B$. longum expressed similar levels of BDNF mRNA as neuroblastoma cells incubated with sera obtained from healthy mice treated with placebo only, suggesting lack of direct stimulation of BDNF production on neural cells by serum metabolites produced or induced by $B$. longum (Fig. 4).

\section{Effect of $B$. longum fermentation medium on enteric neuron excitability}

The perfusion of B. longum FM over enteric neurons decreased their excitability. The electro-responsiveness of neurons (number of action potentials/stimulus) in the control group (Krebs solution, $n=5$ ) was $2.1 \pm 1.1$ and in the neurons perfused with $\mathrm{CM}(n=4)$ was $1.7 \pm 0.9$ (non-significant). However, the electro-responsiveness of neurons perfused with $B$. longum FM $(n=4)$ was only $0.3 \pm 0.5(P=0.02$ vs Krebs and $P=0.03$ vs $\mathrm{CM})$. The instantaneous input resistance was similar in Krebs $(131 \pm 70 \mathrm{M} \Omega)$, B. longum FM (87 \pm 56 $\mathrm{M} \Omega)$ and $\mathrm{CM}(175 \pm 97 \mathrm{M} \Omega$ ) perfused neurons (Fig. 5).

\section{Detection of $B$. longum in feces of mice with DSS colits}

Bifidobacterium longum was not detected in placebotreated mice, with $(n=11)$ or without vagotomy $(n=15)$. In mice treated with $B$. longum, the probiotic was detected in eight out of nine sham-operated mice, and in all 14 vagomized mice (Fig. 6).

\section{DISCUSSION}

We have shown that chronic mild DSS colitis induces anxiety-like behavior in mice, and that the vagus nerve is required for gut-brain communication in this model. Similarly to our recent study with colonic T. muris infection, ${ }^{6}$ administration of the probiotic $B$. longum NCC3001 normalized behavior in mice with DSS colitis without affecting gut inflammation. We did not observe a direct effect of sera from $B$. longumtreated mice on BDNF mRNA expression in neuroblastoma cell cultures, arguing against a direct effect of $B$. longum fermentation products on the CNS. However, we demonstrated that the anxiolytic effect of $B$. longum is dependent on vagus nerve integrity and that the products generated during bacterial fermentation modulate excitability of the enteric neurons in the gut suggesting that enteric neurons might function as gateways to the CNS.

Dextran sodium sulfate colitis is a well-described and validated model of acute and chronic colitis. ${ }^{15}$ We investigated the effect of three cycles of low dose DSS administration on gut inflammation and accompanying changes in mouse behavior. We induced mild chronic colitis as evidenced by low composite histology scores and minimally elevated MPO levels. 
We have previously shown, using a non-invasive parasite $T$. muris, that moderate infectious colitis is associated with anxiety and depression-like behavior and changes in brain biochemistry. ${ }^{6}$ Thus, in the present study we expand those findings to demonstrate that anxiety-like behavior is also present in mice with mild, chemically induced colitis.

Treatment with $B$. longum not only normalized the altered behavior of mice with DSS colitis, but $B$. longum-treated mice showed markedly increased exploratory behavior compared with healthy controls. This effect was largely independent of probiotic activity on gut inflammation, as B. longum administration did not improve inflammation scores or MPO activity. This is in agreement with our previous study, where we demonstrated that $B$. longum was able to improve anxiety-like behavior but had no effect on gut inflammation or circulating cytokines. ${ }^{6}$

We found that vagal nerve integrity is important in the induction of anxiety-like behavior in mice with DSS colitis. This is in contrast with our results in T. muris colitis, where induction of anxiety-like behavior was not vagally mediated. The differential role of the vagus nerve in the two murine models can be explained by the different patterns of inflammation induced: while chronic T. muris infection is exclusively localized to the cecum and colon of mice, 16,17 DSS also affects the small intestine. ${ }^{18,19}$ As the vagus nerve is known to innervate the proximal GI tract with less fibers extending into the large intestine in mice ${ }^{20}$ and the colon being mainly innervated by spinal nerves, ${ }^{21}$ the absence of anxiety-like behavior observed in vagotomized mice subsequently treated with DSS may be due to loss of signaling from the small intestine. To overcome this issue when investigating the role of vagal pathways in $B$. longum action, in the second protocol we performed vagotomy before the third cycle of DSS in mice, in whom the anxiety-like behavior was already established after two cycles. In these mice, treatment with $B$. longum did not ameliorate anxiety-like behavior indicating that the vagus nerve is essential for its anxiolytic effect. Furthermore, the impact of $B$. longum on behavior in healthy mice was also abrogated by vagotomy. Our results thus suggest that vagal pathways are capable of mediating signals that induce both pro and antianxiety effects depending on the nature of the stimulus (i.e. DSS proanxiety vs B. longum anxiolytic).

Brain derived neurotrophic factor is a neurotrophin involved in neural development and plasticity that plays an important role in learning and memory, as well as anxiety and depression. ${ }^{22}$ As $B$. longum has been shown to up-regulate hippocampal expression of BDNF, ${ }^{6}$ we investigated whether this bacterium can directly affect expression of this neurotrophin in neural cells via production of bioactive molecules during fermentation. We used differentiated human neuroblastoma cells and incubated them with sera from healthy control and from $B$. longum-treated mice. We did not observe a significant difference in BDNF mRNA expression, suggesting that a direct effect of $B$. longum systemic metabolites on central BDNF production is unlikely. Further studies are required to determine whether sera from B. longum-treated animals with DSS colitis will produce a similar effect.

Our experiments using an ileal preparation in vitro showed that $B$. longum fermentation products can modify the functional status of enteric neurons in the gut. The firing of action potentials in response to electrical stimulation was greatly decreased in enteric nerves perfused with $B$. longumFMindicating that their excitability was directly modulated by 
probiotic fermentation products. This leads to the hypothesis that the probiotic communicates with the CNS through enteric nerves. The identity of nerves affected by the probiotic needs further study. Previous studies have shown that other probiotic bacteria such as Lactobacillus reuteri were able to prevent hyperexcitability of colonic DRG neurons induced by noxious stimuli ${ }^{23}$ and altered baseline excitability of colonic $\mathrm{AH}$ neurons by inhibiting calcium-dependent potassium channels. ${ }^{24}$ Others have shown that acute intraduodenal administration of Lactobacillus johnsonii can affect renal sympathetic and gastric vagal nerve activity through histaminergic pathways. ${ }^{25}$ Similarly, colonization with the pathogen Campylobacter jejuni was shown to activate brain vagal centers within several hours postinfection. ${ }^{4}$ In our study, vagotomy abolished the effect of $B$. longum on behavior, suggesting that vagal pathways are crucial for the expression of its central effects. However, whether the effects are due to direct stimulation of extrinsic vagal afferent nerve terminals or are secondary to effects on intrinsic enteric nerves requires further investigation. Further studies are also required to determine whether the effects on the enteric neuronal preparation are maintained in the presence of an intact mucosal barrier. We have not performed a detailed analysis of microbial communities before and after administration of B. longum. Thus, it remains to be determined whether some of the behavioral effects observed are associated with changes in the intestinal microbiota.

In summary, $B$. longum NCC3001 normalizes mouse anxiety-like behavior induced by gut chronic inflammation, irrespective of whether this is caused by chronic parasite infection ${ }^{6}$ or chemically induced, as shown in this study. The anxiolytic effects of $B$. longum appear to be independent of immunomodulation or of direct up-regulation of BDNF in neural cells. As $B$. longum has the ability to affect the function of enteric neurons, we hypothesize that its anxiolytic effect is mediated through vagal pathways originating either at the level of the ENS or directly on vagal afferent terminals innervating the gut. Bifidobacterium longum NCC3001 may have a promising therapeutic potential in patients with chronic gut disorders and psychiatric co morbidity. One interesting finding of our study relates to the possible effect of $B$. longum on enhancement of exploratory behavior in healthy mice, and its possible future application as a health supplement. Clinical studies are warranted to determine whether this probiotic has an equivalent effect on mood and general well-being in humans.

\section{Acknowledgments}

This study was supported by CCFC grants to Dr E.F. Verdu, P. Bercik and S.M. Collins and by a grant by Nestle Research Center, and partially by a CIHR grant to J.D. Huizinga. Drs. EFV and PB are recipients of Internal Career Awards by the Department of Medicine, McMaster University.

\section{References}

1. Walker JR, Ediger JP, Graff LA, et al. The Manitoba IBD cohort study: a population-based study of the prevalence of lifetime and 12-month anxiety and mood disorders. Am J Gastroenterol. 2008; 103:1989-97. [PubMed: 18796096]

2. Whitehead WE, Palsson O, Jones KR. Systematic review of the comorbidity of irritable bowel syndrome with other disorders: what are the causes and implications? Gastroenterology. 2002; 122:1140-56. [PubMed: 11910364] 
3. Lyte M, Varcoe JJ, Bailey MT. Anxiogenic effect of subclinical bacterial infection in mice in the absence of overt immune activation. Physiol Behav. 1998; 65:63-8. [PubMed: 9811366]

4. Goehler LE, Gaykema RP, Opitz N, et al. Activation in vagal afferents and central autonomic pathways: early responses to intestinal infection with Campylobacter jejuni. Brain Behav Immun. 2005; 19:334-44. [PubMed: 15944073]

5. Bercik P, Verdú EF, Foster JA, et al. Role of gut-brain axis in persistent abnormal feeding behavior in mice following eradication of Helicobacter pylori infection. Am J Physiol Regul Integr Comp Physiol. 2009; 296:R587-94. [PubMed: 19129375]

6. Bercik P, Verdu EF, Foster JA, et al. Chronic gastrointestinal inflammation induces anxiety-like behavior and alters central nervous system biochemistry in mice. Gastroenterology. 2010; 139:2102-12. [PubMed: 20600016]

7. O'Connor JC, Lawson MA, André C, et al. Lipopolysaccharide-induced depressive-like behavior is mediated by indoleamine 2,3-dioxygenase activation in mice. Mol Psychiatry. 2009; 14:511-22. [PubMed: 18195714]

8. Anisman H, Hayley S, Kelly O, et al. Psychogenic, neurogenic, and systemic stressor effects on plasma corticosterone and behavior: mouse strain-dependent outcomes. Behav Neurosci. 2001; 115:443-54. [PubMed: 11345969]

9. Verma-Gandhu M, Verdu EF, Bercik P, et al. Visceral pain perception is determined by the duration of colitis and associated neuropeptide expression in the mouse. Gut. 2007; 56:358-64. [PubMed: 17018864]

10. Verdú EF, Deng Y, Bercik P, Collins SM. Modulatory effects of estrogen in two murine models of experimental colitis. Am J Physiol Gastrointest Liver Physiol. 2002; 283:G27-36. [PubMed: 12065288]

11. Verdu EF, Bercik P, Bergonzelli GE, et al. Lactobacillus paracasei normalizes muscle hypercontractility in a murine model of postinfective gut dysfunction. Gastroenterology. 2004; 127:826-37. [PubMed: 15362038]

12. Garzon DJ, Fahnestock M. Oligomeric amyloid decreases basal levels of brain-derived neurotrophic factor (BDNF) mRNA via specific down-regulation of BDNF transcripts IV and V in differentiated human neuroblastoma cells. J Neurosci. 2007; 27:2628-35. [PubMed: 17344400]

13. Serres F, Carney SL. Nicotine regulates SH-SY5Y neuroblastoma cell proliferation through the release of brain-derived neurotrophic factor. Brain Res. 2006; 1101:36-42. [PubMed: 16790237]

14. Rouge C, Goldenberg O, Ferraris L, et al. Investigation of the intestinal microbiota in preterm infants using different methods. Anaerobe. 2010; 16:362-70. [PubMed: 20541022]

15. Wirtz S, Neufert C, Weigmann B, Neurath MF. Chemically induced mouse models of intestinal inflammation. Nat Protoc. 2007; 2:541-6. [PubMed: 17406617]

16. Panesar TS, Croll NA. The location of parasites within their hosts: site selection by Trichuris muris in the laboratory mouse. Int J Parasitol. 1980; 10:261-73. [PubMed: 7451019]

17. Mahida YR. Host-parasite interactions in rodent nematode infections. J Helminthol. 2003; 77:12531. [PubMed: 12756066]

18. Ohtsuka Y, Sanderson IR. Dextran sulfate sodium-induced inflammation is enhanced by intestinal epithelial cell chemokine expression in mice. Pediatr Res. 2003; 53:143-7. [PubMed: 12508094]

19. Yazbeck R, Howarth GS, Butler RN, Geier MS, Abbott CA. Biochemical and histological changes in the small intestine of mice with dextran sulfate sodium colitis. J Cell Physiol. 2011 Feb 23. [Epub ahead of print]. doi: 10.1002/jcp.22682

20. Andrews PL, Sanger GJ. Abdominal vagal afferent neurones: an important target for the treatment of gastrointestinal dysfunction. Curr Opin Pharmacol. 2002; 2:650-6. [PubMed: 12482726]

21. Vizzard MA, Brisson M, de Groat WC. Transneuronal labeling of neurons in the adult rat central nervous system following inoculation of pseudorabies virus into the colon. Cell Tissue Res. 2000; 299:9-26. [PubMed: 10654066]

22. Deltheil T, Guiard BP, Cerdan J, et al. Behavioral and serotonergic consequences of decreasing or increasing hippocampus brain-derived neurotrophic factor protein levels in mice. Neuropharmacology. 2008; 55:1006-14. [PubMed: 18761360] 
23. Ma X, Mao YK, Wang B, Huizinga JD, Bienenstock J, Kunze W. Lactobacillus reuteri ingestion prevents hyperexcitability of colonic DRG neurons induced by noxious stimuli. Am J Physiol Gastrointest Liver Physiol. 2009; 296:G868-75. [PubMed: 19179624]

24. Kunze WA, Mao YK, Wang B, et al. Lactobacillus reuteri enhances excitability of colonic AH neurons by inhibiting calcium-dependent potassium channel opening. J Cell Mol Med. 2009; 13:2261-70. [PubMed: 19210574]

25. Tanida M, Yamano T, Maeda K, Okumura N, Fukushima Y, Nagai K. Effects of intraduodenal injection of Lactobacillus johnsonii La1 on renal sympathetic nerve activity and blood pressure in urethane-anesthetized rats. Neurosci Lett. 2005; 389:109-14. [PubMed: 16118039] 

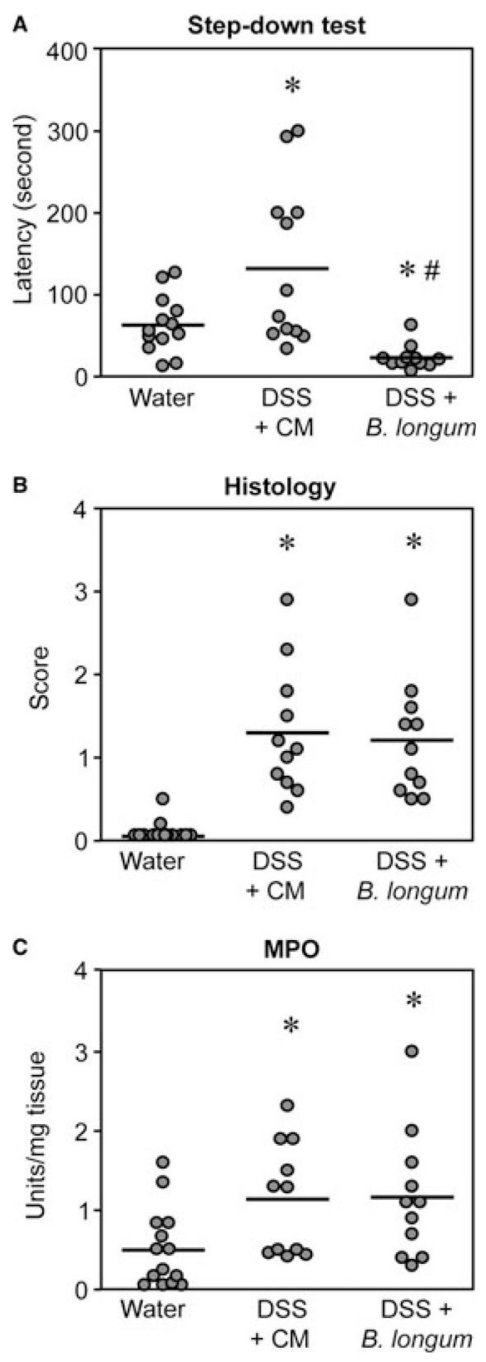

Figure 1.

Anxiolytic effect of Bifidobacterium longum in dextran sodium sulfate (DSS) colitis is independent of its anti-inflammatory activity. (A) Latency to step down from the elevated platform in control (water, $n=13)$, DSS $+\mathrm{CM}(n=12)$ and DSS + B. longum treated $(n=$ 11) mice. (B) Histology composite score of colonic inflammation in control, DSS + CM and DSS + B. longum treated mice. (C) Myeloperoxidase activity in colon tissues of control, $\mathrm{DSS}+\mathrm{CM}$ and DSS $+B$. longum treated mice. Individual values are shown. Bars indicate mean $\left(* P<0.05\right.$ vs water, ${ }^{*} P<0.05$ vs DSS-CM treated mice). 


\section{Step-down test}

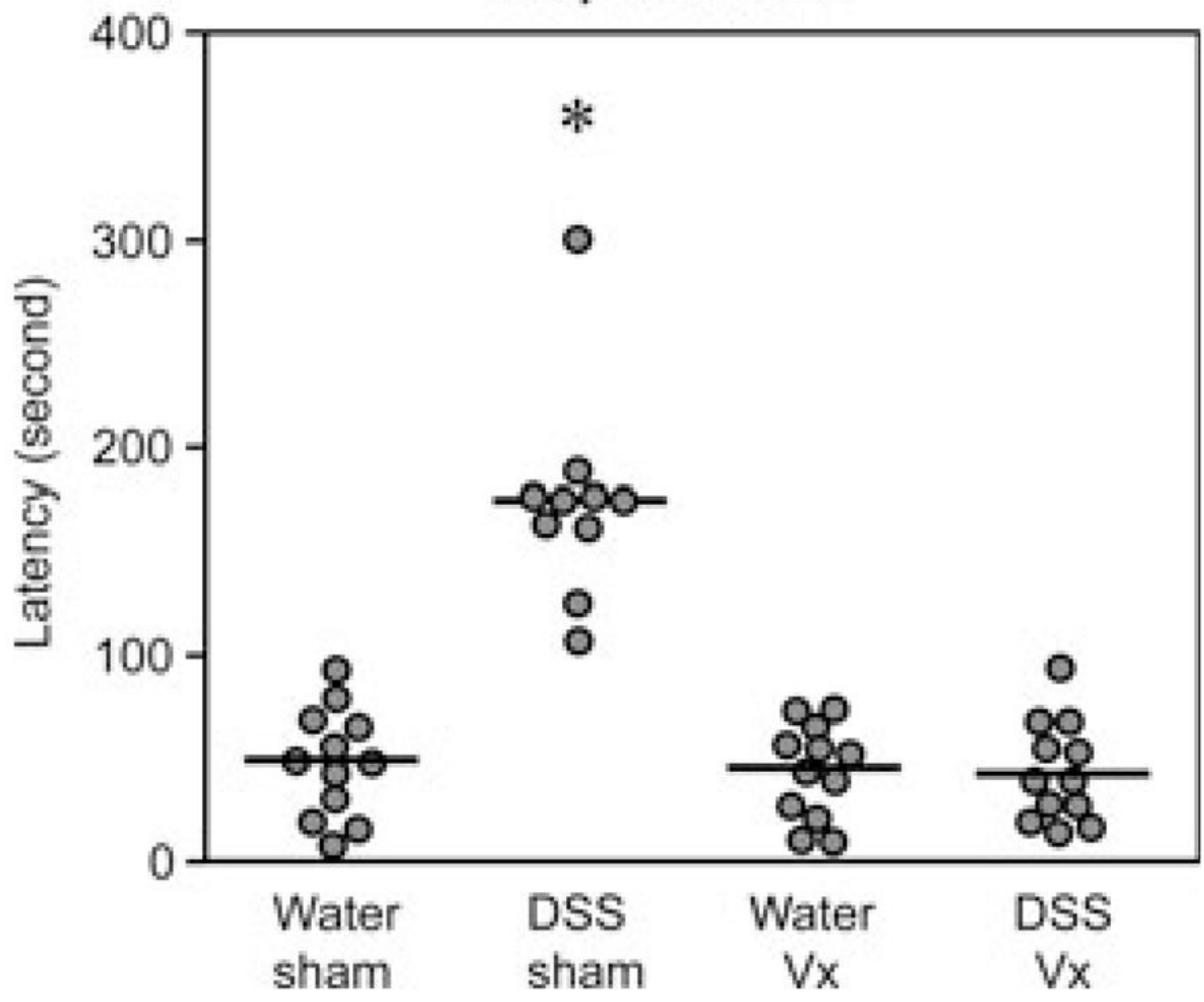

Figure 2.

Role of vagus in dextran sodium sulfate (DSS)-colitis induced anxiety-like behavior. Latency to step down from the elevated platform in sham operated controls (water, $n=12$ ), sham operated mice treated with DSS $(n=10)$, vagotomized controls (water Vx, $n=12)$ and vagotomized mice treated with DSS (DSS Vx, $n=12$ ) mice. Vagotomy was performed before the first cycle of DSS. Individual values are shown. Bars indicate mean $(* P<0.05$ vS all other groups). 

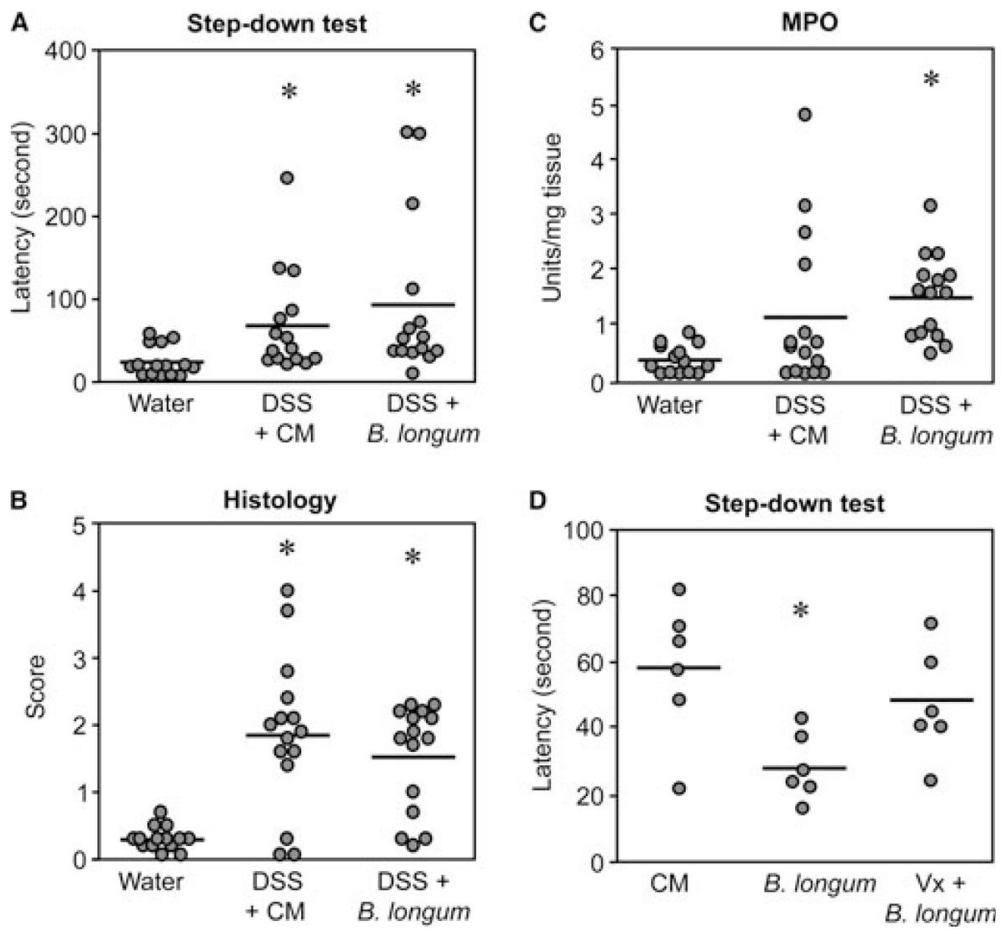

Figure 3.

Anxiolytic effect of Bifidobacterium longum during dextran sodium sulfate (DSS) colitis requires the integrity of the vagus. (A) Latency to step down from the elevated platform in control vagotomized mice (water, $n=14)$, vagotomized mice treated with DSS + CM ( $n=$ $15)$ and vagotomized mice treated with DSS $+B$. longum $(n=15)$. Vagotomy was performed before the third cycle of DSS. (B) Histology composite score in control vagotomized mice (water), vagotomized mice treated with DSS + CM and vagotomized mice treated with DSS and B. longum. (C). Myeloperoxidase activity in colon tissue of in control vagotomized mice (water), vagotomized mice treated with DSS + CM and vagotomized mice treated with DSS and B. longum. Individual values are shown. (D) Latency to step down from the elevated platform in control mice (water, $n=6$ ), mice treated with $B$. longum $(n=6)$ and vagotomized mice $(\mathrm{Vx})$ treated with $B$. longum $(n=15)$. Bars indicate mean $(* P<0.05$ vs water $)$. 


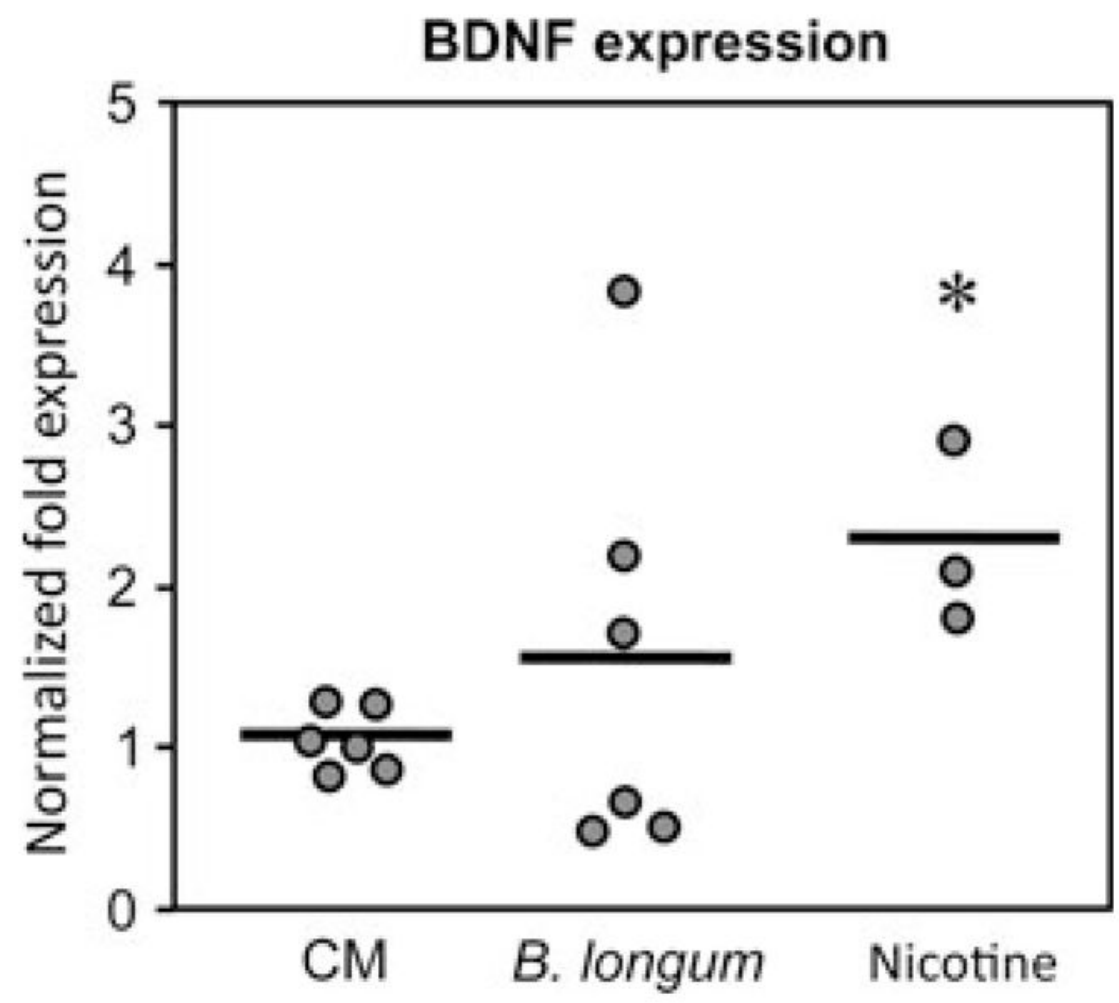

Figure 4.

Bifidobacterium longum fermentation products do not affect basal level of BDNF mRNA expression in neural cells. BDNF mRNA expression in human SH-SY5Y neuroblastoma cells exposed to serum from either CM (placebo) or B. longum-treated mice. Nicotine was used as a positive control. Each value represents the average of a duplicate sample from a single culture, horizontal bar indicates group mean. BDNF data were normalized to GAPDH expression. 
A

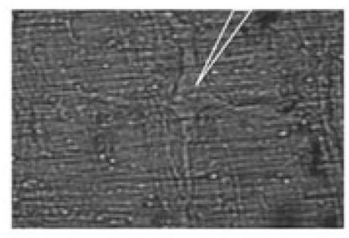

C
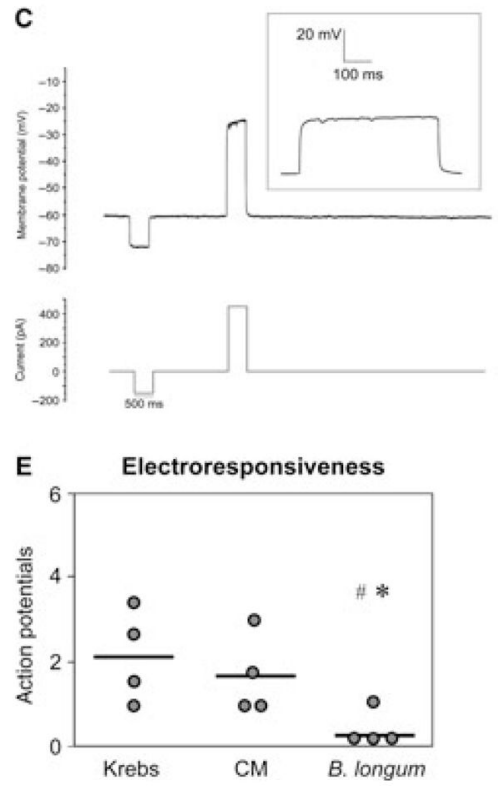

Figure 5.

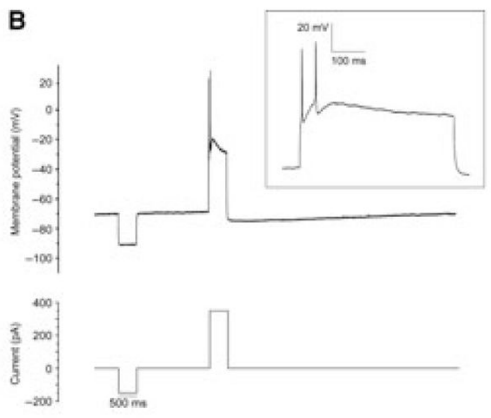

D

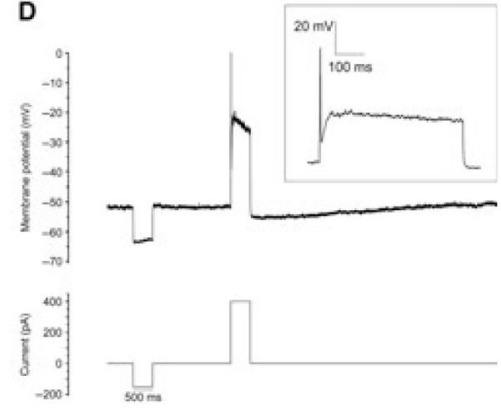

Bifidobacterium longum fermented mediumdecreases the excitability of enteric neurons. (A)

Representative image of an individual neuron being impaled with an intracellular microelectrode (in white). (B-D) Intracellular microelectrode recording showing action potentials discharged. Inset images show injection of a $500 \mathrm{~ms}$ duration current pulse with various intensities (pA). (B) Enteric neuron exposed to Krebs only. This neuron fired two action potentials in response to a supra-threshold current pulse set at threshold (350 pA) plus 50 millivolts. (C) Enteric neuron exposed to B. longum FM. No action potentials were discharged from this neuron after the injection of a $500 \mathrm{~ms}$ duration current pulse with 450 pA intensity. (D) Enteric neuron exposed to control (CM) only. This neuron fired one action potential in response to a supra-threshold pulse set at threshold plus 50 millivolts. (E) Electroresponsiveness of enteric nerves perfused with Krebs, CM or B. longum FM. Individual values are shown. Bars indicate mean ( ${ }^{*} P<0.05$ vs $\mathrm{Krebs},{ }^{\#} P<0.05$ vs $\left.\mathrm{CM}\right)$. 


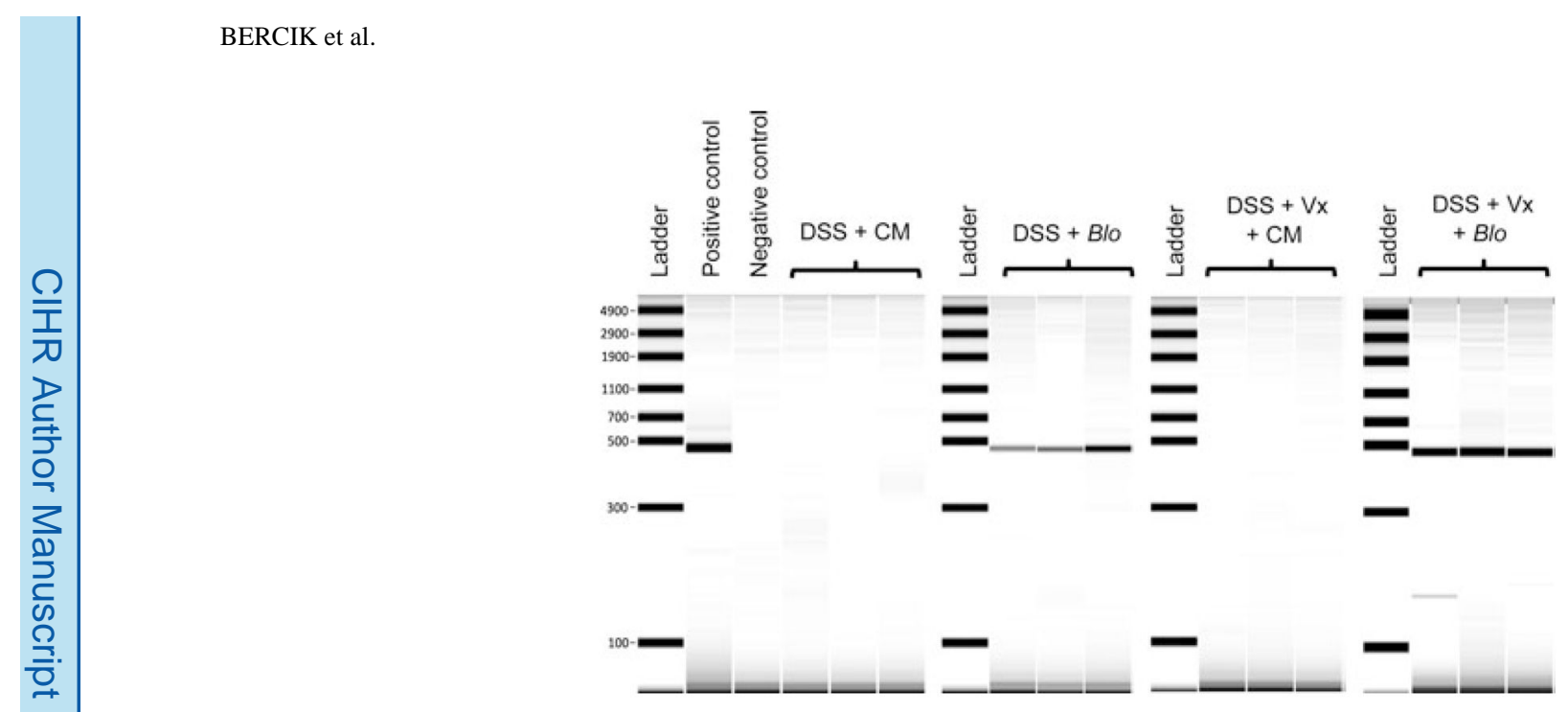

Page 17

Figure 6.

Detection of Bifidobacterium longum in feces of mice with dextran sodium sulfate (DSS) colitis. Representative PCR gels of B. longum in mice trated with CM or B. longum, with or without vagotomy $(\mathrm{Vx})$. 